\title{
Cosine Modulated and Offset QAM Filter Bank Multicarrier Techniques: A Continuous-Time Prospect
}

\author{
Behrouz Farhang-Boroujeny and Chung Him (George) Yuen \\ ECE Department, University of Utah, UT 84112, USA \\ Correspondence should be addressed to Behrouz Farhang-Boroujeny, farhang@ece.utah.edu \\ Received 11 May 2009; Revised 23 September 2009; Accepted 14 December 2009 \\ Academic Editor: Pierre Siohan
}

Copyright () 2010 B. Farhang-Boroujeny and C. H. (George) Yuen. This is an open access article distributed under the Creative Commons Attribution License, which permits unrestricted use, distribution, and reproduction in any medium, provided the original work is properly cited.

\begin{abstract}
Prior to the discovery of the celebrated orthogonal frequency division multiplexing (OFDM), multicarrier techniques that use analog filter banks were introduced in the 1960s. Moreover, advancements in the design of perfect reconstruction filter banks have led to a number developments in the design of prototype digital filters and polyphase structures for efficient implementations of the filter bank multicarrier (FBMC) systems. The main thrust of this paper is to present a tutorial review of the classical works on FBMC systems and show that some of the more recent developments are, in fact, reinventions of multicarrier techniques that have been developed prior of the era of OFDM. We also review the recent novel developments in the design of FBMC systems that are tuned to cope with fast fading wireless channels.
\end{abstract}

\section{Introduction}

Orthogonal frequency division multiplexing (OFDM) is the most dominant technology that has been researched and has been deployed for broadband wireless communications. OFDM is attractive because of a number of advantages that it offers. First, orthogonality of subcarrier channels allows trivial equalization; one scalar gain per subcarrier. Second, closely spaced orthogonal subcarriers partition the available bandwidth into a collection of narrow subbands. Adaptive modulation schemes are then applied to sub-bands to maximize bandwidth efficiency/transmission rate. Third, the very special structure of OFDM symbols simplifies the tasks of carrier and symbol synchronizations. These points are well understood and documented in the literature $[1,2]$.

More recent works propose extending the use of OFDM to multiple access applications. Multiple access OFDM, or orthogonal frequency division multiple access (OFDMA), has recently been proposed in a number of standards and proprietary waveforms (e.g., [3]). Some particular forms of OFDMA have also been proposed for cognitive radio systems [4]. In OFDMA, a subset of the subcarriers is allocated to each user node in a network. These users signals must be synchronized at the receiver input to prevent intercarrier interference. OFDMA works well in the network downlink of a base station, since all of the subcarriers are transmitted from the same base station and, thus, can easily be synchronized. However, synchronization is not trivial in the network uplink where a number of nodes are transmitting separately. For OFDMA to work well in this scenario, the signals from various nodes must be synchronized at the base station, that is, they should be received as a set of orthogonal signals. Since, in practice, perfect synchronization may not be possible, additional signal processing steps have to be taken to minimize interference among signals from different nodes. Such steps add significant complexity to an OFDMA receiver; see [5] and the references therein. The problem is worse in a cognitive radio setting where both primary (non-cognitive nodes) and secondary users (cognitive nodes) transmit independently and may be based on different standards. Therefore, the existing OFDMA may not be able to satisfactorily address the needs of efficient use of spectra in the next generation of communication networks.

Clearly, the above problem could be greatly alleviated if the filters that synthesize the subcarrier signals had small side-lobes. An interesting, but apparently not widely 
understood, fact is that the first multicarrier techniques which were developed before the invention of OFDM used filter banks for synthesis and analysis of multicarrier signals. Such filter banks can be designed with small side-lobes, thus, are ideal choice in multiple access and cognitive radio applications [6]. The first proposal came from Chang [7], who presented the conditions required for signaling a parallel set of pulse amplitude modulated (PAM) symbol sequences through a bank of overlapping filters within a minimum bandwidth. To transmit PAM symbols in a bandwidth efficient manner, Chang's signaling is based on staggering a number of overlapping vestigial side-band (VSB) modulated signal sequences. Saltzberg [8], extended the idea and showed how the Chang's method could be modified for transmission of quadrature amplitude modulated (QAM) symbols, in a double side-band modulated format. Efficient digital implementation of Saltzberg's multicarrier system through polyphase structures first introduced by Bellanger et al. $[9,10]$, was studied by Hirosaki $[11,12]$, and was further developed by others [13-21]. Both Chang's and Saltzberg's methods belong to a class of multicarrier techniques that may be referred to as filter bank multicarrier (FBMC) systems.

The pioneering work of Chang [7], on the other hand, has received less attention within the signal processing community. Those who have cited [7], have only acknowledged its existence without presenting much details, for example $[11,16,19,22]$. For instance, Hirosaki who has extensively studied and developed digital structures for implementation of Saltzberg's method, $[11,16]$, has made a brief reference to Chang's method and noted that since it uses VSB modulation and thus its implementation require a Hilbert transformation, it is more complex than that of Saltzberg's method. He thus proceeds with a detail discussion and development of multirate structures for the Saltzberg's method only. On the other hand, a vast literature in digital signal processing has studied a class of multicarrier systems that has been referred to as discrete wavelet multitone (DWMT). The initial works on DWMT are [23-25]. In the period of 1995 to 2003 a fair number of contributions from various authors appeared in the literature, for example [26-30]. Reference [30], in particular, did a thorough study of DWMT and noted that this method operates based on cosine modulated filter banks which were extensively developed in the 1980's in the context of compression techniques [31]. The fact that DWMT uses cosine modulated filter banks has also been acknowledged by other authors, for example [28]. Reference [30] also greatly simplified the equalizer structure that was originally proposed in [23-25] and widely adopted by others. Moreover, [30] noted that a DWMT signal is synthesized by aggregating a set of VSB modulated PAM signal sequences. However, most of the works on DWMT (including [30]) have made no direct reference to Chang's method. In other words, the Chang's multicarrier method was re-invented, with a strong multirate signal processing flavor, in the 1990's. Part of our attempt in this paper is to show this very important relationship between what has been done over 40 years ago, and the independent developments on DWMT/cosine modulated multicarrier techniques that have been developed in more recent years. We also hope that the tutorial treatment of the Chang's method in this paper will facilitate a more in-depth understanding of the DWMT/cosine modulated multicarrier literature. Another important point to note is that although DWMT was originally developed with DSL applications in mind, it was never adopted in any of DSL standards. However, DWMT has recently found its way to power line communications (PLC) that share a very similar environment to that of DSL [32].

It is also interesting to note that the researchers who studied filter banks developed a class of filter banks which were called modified DFT (MDFT) filter bank [33]. Careful study of MDFT reveals that this, although done independently, is in effect a reformulation of Saltzberg's filter bank in discrete-time and with emphasis on compression/coding. The literature on MDFT begins with the pioneering works of Fliege [34], and later has been extended by others, for example [35-38].

As a final note in this introductory section, we wish to bring the attention of reader to various terminologies that have appeared in the literature related to Chang's and Saltzberg's MCFB methods and many further extensions that have been made by others. In the pioneering works of Chang [7] and Saltzberg [8] no specific name has been given to the multicarrier modulation types that they introduce, except that Chang notes PAM symbols are transmitted via its signaling method and Saltzberg notes that how QAM symbols can be transmitted with the same bandwidth efficiency. Even the fact that Chang's subcarrier modulation is VSB has not been explicitly noted in his paper [7]. Apparently, the name staggered QAM was used for the type of modulation suggested in [8], for the first time, in [39]. Later, Hirosaki [12] used the terminology orthogonally multiplexed QAM (OQAM). OQAM was later referred to as OFDM-OQAM by many authors, for example [13-15, 1721], with the acronym OQAM standing for offset QAM, reflecting the fact that the in-phase and quadrature of each QAM symbol are time offset with respect to each other. A few others have named it pulse-shaped OFDM [4047]. These use of different terminologies in parallel with the independent introduction of MDFT, which is based on the same fundamental principles as OQAM, has made the literature on FBMC techniques somewhat blurred [48], and thus confusing to any novice who wishes to begin a research in this area.

The same is true for Chang's method and the independent, but related, works that have been published later. Among these [25], that received a significant level of attention (see [30] and the references therein), independently (but, effectively) presented Chang's modulation scheme under the name discrete wavelet multitone (DWMT). The name DWMT is somewhat confusing here as the proposed method in fact uses cosine modulated filter banks for which the use of the terminology wavelet is a misnomer. Cosine modulated filter banks belong to the class of uniform filter banks, meaning that all subbands have the same width. Wavelets, on the other hand, are referred to filter banks whose subband widths increase exponentially with the respective carrier/center frequencies. The adjective dyadic is often used to address this property of wavelets. It is 
also interesting to note that there exists another class of multicarrier techniques that are based on true wavelets (i.e., wavelets with dyadic bandwidths), for example, see [49]. Moreover, it is worth noting that the IEEE P1901 working group who has adopted a DWMT type modulation for part of PLC standard, has called it wavelet-OFDM. On the other hand, some authors have preferred the name cosine modulated filter bank OFDM (CMFB-OFDM). In [50], where a more thorough study of DWMT/CMFBOFDM to VDSL has been presented, the shorter name cosine-modulated multitone (CMT) has been proposed, following the terminology filtered multitone (FMT) [5153], another FBMC candidate that was proposed (but was never adopted) in VDSL standard. In this paper, we use CMT when reference is made to the Chang's method (and its extensions). We also introduce and use the terminology staggered-modulated multitone (SMT) for the Saltzberg's method (and its extensions).

In this paper, we first present a novel tutorial review of Chang's and Saltzberg's FBMC methods with the goal of making these classical works more accessible to the signal processing community. These are presented in Sections 2 and 3 , respectively. Similarities and differences of CMT and SMT are discussed in Section 4. In Section 5 further development that has been made on extensions on Chang's and Saltzberg's methods are discussed. The emphasis in this section is on the design of prototype filters for CMT and SMT. A brief review of equalization of CMT and SMT systems is presented in Section 6. The concluding remarks are made in Section 7.

Even though, any modern implementation of a CMT or SMT system will be in discrete-time, the derivations in this paper are in terms of continuous-time signals and systems. The choice of the continuous-time formulation here simplifies the derivations and will also provide more insight to the fundamental properties of both CMT and SMT as well as their similarities and differences. We believe our approach also provides a meaningful intuitive understanding of the extension of CMT and SMT that are discussed in Section 5.

\section{Cosine Modulated Multitone (CMT)}

In CMT, a number of parallel streams of PAM data symbols are transmitted through a set of vestigial side-band (VSB) subcarrier channels. Moreover, the subcarrier channels are minimally spaced to maximize the bandwidth efficiency of the system. To explain what constitutes to the minimally spaced subcarrier channels, we recall that the minimum bandwidth for a transmission rate of $R=1 / T$ QAM symbols per second, where $T$ is symbol spacing in seconds, is $B=$ $1 / \mathrm{T} \mathrm{Hz},[54]$. This concept is demonstrated in Figure 1, where the magnitude response of a Nyquist filter with an excess bandwidth of $\alpha / 2 T$ is presented. Clearly, the minimum bandwidth is achieved when $\alpha=0$. In CMT, where data symbols are PAM, noting that each PAM symbol is equivalent to one half of a QAM symbol, one may argue that the minimum bandwidth for transmission of $R=1 / T$ PAM symbols per second is $B=1 / 2 T \mathrm{~Hz}$. To further clarify this point and pave the way for development of a CMT system, we continue with an introduction to a VSB channel.

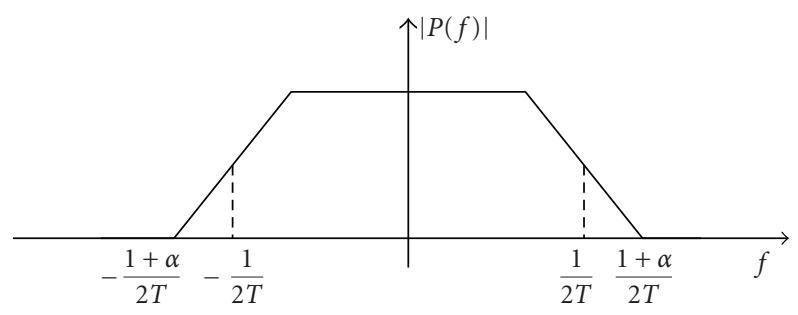

FIGURE 1: Magnitude response of a Nyquist filter with an excess bandwidth of $\alpha / T$. Note that $P(f)$ has the total banwidth $(1+$ $\alpha) / 2 T-(-(1+\alpha) / 2 T)=(1+\alpha) / T$, and the minimum bandwidth $1 / T$ is achieved when $\alpha=0$.

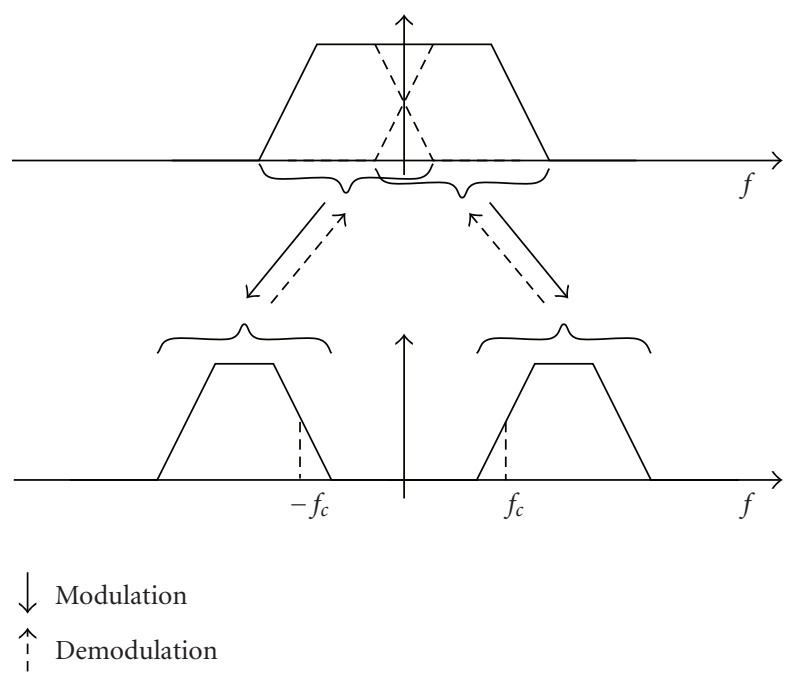

Figure 2: Vestigial side-band modulation and demodulation. The figure depicts the signals spectra.

2.1. Vestigial Side-Band Modulation. Figure 2 presents the process of taking a baseband signal, modulating it to a VSB channel, and demodulating the modulated signal back to the baseband. As shown, for modulation, this process divides the spectrum of the baseband signal in two parts, takes one part to modulate a complex sine-wave at the positive frequency $f_{c}$ (i.e., multiply by $e^{j 2 \pi f_{c} t}$ ) and the other part to modulate a complex sine-wave at the negative frequency $-f_{c}$ (i.e., multiply by $\left.e^{-j 2 \pi f_{c} t}\right)$. Demodulation is performed by reversing these steps.

Next, we explain the above steps in a form closely related to CMT modulation. Consider the communication system shown in Figure 3(a). The input signal $s(t)$ is an impulse train corresponding to a PAM data stream $s[n]$ at the rate of $1 / T$, viz.,

$$
s(t)=\sum_{n=-\infty}^{\infty} s[n] \delta(t-n T) .
$$

In Figure $3, h(t)$ is a lowpass filter whose bandwidth is one half of the bandwidth of a typical pulse-shaping filter which one would use for transmission of the data symbols $s[n]$ at a rate of $1 / T$. We further assume that $h(t)$ is a zero-phase filter, that is, satisfies the symmetry condition $h(-t)=h(t)$ 


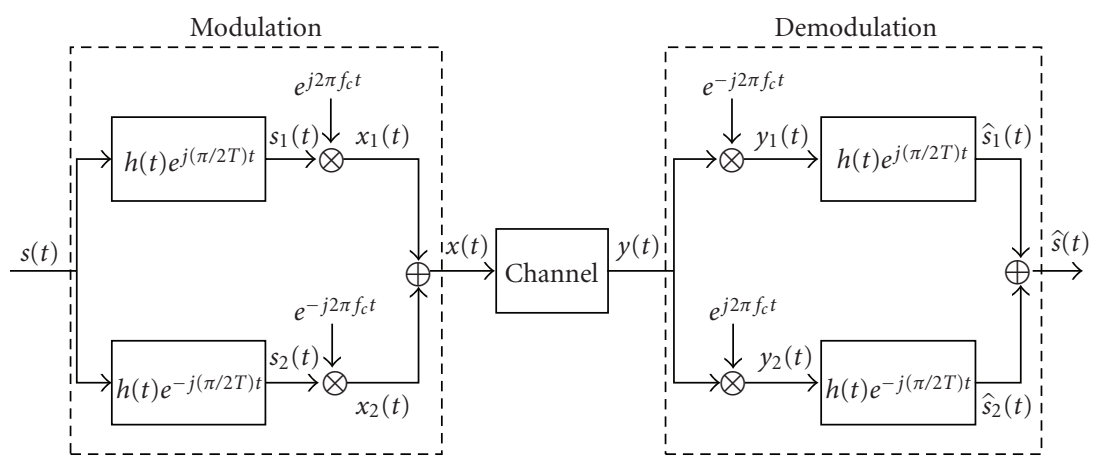

(a)

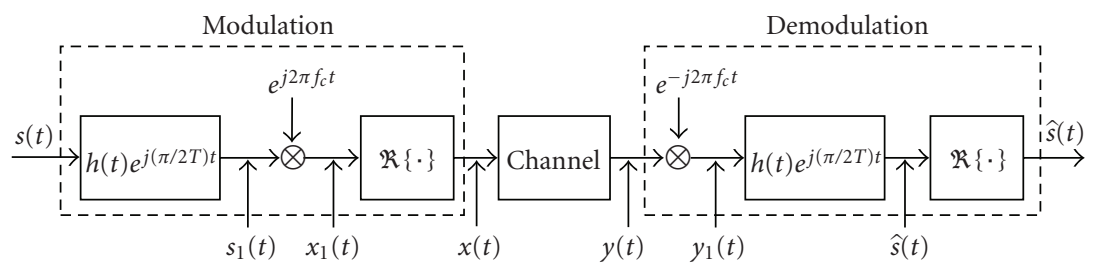

(b)

Figure 3: Vestigial side-band modulation and demodulation. (a) Detailed block diagram. (b) Simplified block diagram.

and $h(t)$ is a real function of time. Hence, the same filter is used at the receiver as a matched pair to the transmit filter $h(t)$. However, we note that in the original work of Chang, [7], this restriction is not imposed on $h(t)$. Nevertheless, in practice, when filters are realized digitally, the use of zero-phase/symmetric filters may be the most useful case. Moreover, most of the derivations that follow become trivial when $h(t)$ is symmetric. We will make some comments on the more general pulse-shaping filters that have been proposed in [7] later; see Section 2.3.

The filter $h(t) e^{j(\pi / 2 T) t}$ in the upper-left branch of Figure 3(a) is a modulated version of $h(t)$ that is centered at $f=1 / 4 T$ (note that $\pi / 2 T=2 \pi \times(1 / 4 T)$ ). Similarly, the filter in the lower-left branch of Figure $3(a)$ is a modulated version of $h(t)$ that is centered at $f=-1 / 4 T$. Accordingly, the signals $s_{1}(t)$ and $s_{2}(t)$ are the upper- and lower-side bands of a baseband signal. These signals are further modulated to the carrier frequencies $f_{c}$ and $-f_{c}$ to obtain the signals $x_{1}(t)$ and $x_{2}(t)$, respectively. The transmit signal $x(t)$ is obtained by adding $x_{1}(t)$ and $x_{2}(t)$. On the other hand, the received signal $y(t)$ is demodulated to obtain the upper- and lowerside baseband signals $\hat{s}_{1}(t)$ and $\hat{s}_{2}(t)$, respectively. These are then added to obtain the output $\hat{s}(t)$ whose samples, when taken at a correct timing phase, are estimates of the transmitted data symbols $s[n]$. When channel is ideal, that is, $y(t)=x(t)$, these estimates are accurate. The presence of channel noise, clearly, results in some unrecoverable error in the estimates. However, if the channel is flat fading, that is, is characterized by a flat gain, a single-tap equalizer with a complex-valued tap-weight equal to the inverse of the channel gain can be used to combat the channel distortion. Some general comments on the equalizers in FBMC systems will be presented later in Section 6 .
Next, we note that $s(t), h(t), x(t), y(t)$, and $\hat{s}(t)$ are realvalued signals. We take advantage of this fact to simplify the transceiver structure of Figure 3(a). The fact that $s(t)$ is realvalued implies that $x_{1}(t)$ and $x_{2}(t)$ are complex conjugates of each other and, thus, $x(t)=2 \mathfrak{R}\left\{x_{1}(t)\right\}$. This means, in Figure 3(a), the branch that leads to $x_{2}(t)$ may be removed. This results in the left-half side of the simplified block diagram presented in Figure 3(b). Similarly, the fact that $y(t)$ is real-valued can be used to simplify the demodulation part of Figure 3(a) as in Figure 3(b).

Next, we take a closer look at $h(t)$ and discuss how it should be selected in order to result in an intersymbol interference (ISI) free equivalent baseband channel. Assuming an ideal channel between the transmit and receive antennas, the equivalent baseband channel, [54], from $s(t)$ to $\widehat{s}(t)$ has the impulse response

$$
\begin{aligned}
g(t) & =\mathfrak{R}\left\{h(t) e^{j(\pi / 2 T) t} \star h(t) e^{j(\pi / 2 T) t}\right\} \\
& =\Re\left\{e^{j(\pi / 2 T) t} \int_{-\infty}^{\infty} h(\tau) h(t-\tau) d \tau\right\},
\end{aligned}
$$

where $\star$ denotes convolution. Letting $\int_{-\infty}^{\infty} h(\tau) h(t-\tau) d \tau=$ $p(t),(2)$ simplifies to

$$
g(t)=p(t) \cos \left(\frac{\pi}{2 T} t\right)
$$

We recall that for ISI free transmission, $g(t)$ has to be a Nyquist pulse with zero crossings at the points $t=$ $n T$, for all nonzero integer values of $n$. Hence, here, ISI free transmission through the VSB channel is achieved, if $g(n T)=0$, when $n$ is any non-zero integer. To proceed, we first note that $\cos ((\pi / 2 T) n T)=0$ for odd values of $n$. This 
implies that for $g(t)$ to be a Nyquist pulse, it is sufficient that $p(n T)$ be equal to zero at non-zero even values of $n$. This means $p(t)$ has to be a Nyquist pulse with zero-crossings at the interval $2 T$. In other words, $p(t)$ has to be a Nyquist pulse that we would use if the data rate was $0.5 / T$, that is, one half of the rate of $s[n]$. This, in turn, implies that $h(t)$ should be a symmetric square-root Nyquist filter designed for a data rate of $0.5 / T$. This result is in line with the fact that the bandwidth of VSB is one half of that of a double-side band (DSB) channel.

2.2. Aggregating VSB Subcarrier Channels. A CMT signal is obtained by adding a number of VSB modulated signals. As noted earlier, to maximize the bandwidth efficiency of the system, the VSB subcarrier channels across frequency should be spaced at $0.5 / T$. Also, to control and avoid intercarrier interference (ICI) between different subcarrier channels, it is assumed that the underlying filter bank is designed such that only adjacent channels overlap. Then, the ICI among adjacent channels, as shown below, is resolved by introducing a $\pi / 2$ phase shift among each pair of adjacent subcarrier channels.

Figure 4 presents the block diagram of a CMT transceiver. The data signals $s_{0}(t)$ through $s_{N-1}(t)$ are data symbol signals that are defined as in (1). The receiver outputs, $\hat{s}_{k}[n]$, are the detected PAM symbols. By inspection of Figure 4, one finds that each subcarrier channel, say, from the input $s_{k}(t)$ to the output $\hat{s}_{k}[n]$, is similar to the single channel case shown in Figure 3(b). Thus, ISI free communication is established by choosing $h(t)$ to be a square-root Nyquist filter that is designed for a data rate $0.5 / T$. Also, note that the multiplications by $1, \quad e^{j((\pi / T) t+\pi / 2)}, \ldots, e^{j(N-1)((\pi / T) t+\pi / 2)}$ are effectively modulators that organize the subcarrier channels at the center frequencies $0, \pi / T, \ldots, \pi(N-1) / T$. This means the spacing between the adjacent subcarriers is $\pi / T$ radian per second or, equivalently $0.5 / \mathrm{T} \mathrm{Hz}$.

To study the ICI between a pair of adjacent channels, consider the impulse response between $s_{k+1}(t)$ and the point prior to the sampler at the $k$ th output of the receiver. This is obtained by direct inspection of Figure 4, and following the same derivations that led to (3), as

$$
\begin{aligned}
g_{1}(t)= & \Re\left\{h(t) e^{j((3 \pi / 2 T) t+\pi / 2)} \star h(t) e^{j(\pi / 2 T) t}\right\} \\
= & \Re\left\{e^{j((\pi / 2 T) t+(\pi / 2))} \int_{-\infty}^{\infty} h(\tau) e^{j(\pi / T) \tau} h(t-\tau) d \tau\right\} \\
= & -\sin \left(\frac{\pi}{2 T} t\right) \int_{-\infty}^{\infty} h(\tau) h(t-\tau) \cos \left(\frac{\pi}{T} \tau\right) d \tau \\
& -\cos \left(\frac{\pi}{2 T} t\right) \int_{-\infty}^{\infty} h(\tau) h(t-\tau) \sin \left(\frac{\pi}{T} \tau\right) d \tau .
\end{aligned}
$$

We are interested in the sample values of $g_{1}(t)$ at the time instants $n T$, for all integer values of $n$.

For an even value of $n=2 k$, one finds that $\sin ((\pi / 2 T) 2 k T)=\sin (k \pi)=0$ and, thus, (4) reduces to

$$
g_{1}(2 k T)=(-1)^{k+1} \int_{-\infty}^{\infty} h(\tau) h(2 k T-\tau) \sin \left(\frac{\pi}{T} \tau\right) d,
$$

where we have noted that $\cos (k \pi)=(-1)^{k}$. Applying a change of variable $\tau$ to $k T+\tau$ in (5), we get

$$
g_{1}(2 k T)=-\int_{-\infty}^{\infty} h(k T+\tau) h(k T-\tau) \sin \left(\frac{\pi}{T} \tau\right) d=0,
$$

where the second identity follows since the expression under the integral is an odd function of $\tau$. Following a similar procedure, one also finds that $g_{1}(n T)=0$ for odd values of $n$. These results show that there is no ICI between a pair of adjacent subcarrier channels $k$ and $k+1$.

2.3. More Relaxed Forms of $h(t)$. To keep the derivations simple, so far, we have assumed that $h(t)$ is a symmetric (i.e., zero-phase) square-root Nyquist filter. The pioneering work of Chang, [7], derives the necessary and sufficient constraints that should be imposed on $h(t)$, assuming that at the receiver the matched pair of $h(t)$, that is, $h(-t)$, is used for the signal analysis. The Chang's constraints, if given in terms of the Fourier transform of $h(t), H(f)$, and assuming an ideal channel, are the followings:

(1) For practical reasons, it is assumed that $h(t)$ is a real function of time. Accordingly, $|H(f)|$ is an even function of $f$ and $\angle(H(f))$ is an odd function of $f$.

(2) To guarantee ISI free transmission over each subcarrier channel, $p(t)=h(t) \star h(-t)$ must be a Nyquist pulse with regular zero crossings at the intervals of $2 T$. In the frequency domain, this is equivalent to

$$
\sum_{k=-\infty}^{\infty} P\left(f-\frac{k}{2 T}\right)=1
$$

and we may notice that $P(f)=H(f) H^{*}(f)=$ $|H(f)|^{2}$. Note that the symmetry assumption $h(t)=$ $h(-t)$, made previously, implies that $H(f)$ is a real function of $f$, that is, it has zero phase across the frequency. However, since $P(f)=H(f) H^{*}(f)$, the constraint (7) does not require $H(f)$ to be a zero phase filter. In fact, if the only concern is ISI free transmission, $H(f)$ may have any arbitrary phase. In other words, the symmetry assumption $h(t)=h(-t)$ is not necessary, even though some designs in the past have made this assumption for convenience, for example [55].

(3) To guarantee ICI free transmission across each pair of adjacent subcarrier channels, $\angle(H(f))$, in addition to odd symmetry with respect to the point $f=0$ (mentioned above), should also be odd symmetric with respect to the points $f= \pm 1 / 4 T$. Moreover, an additional phase shift of $\pi / 2$ must be introduced between each pair of adjacent subcarrier channels.

(4) The derivations in [7], and also what have been presented so far in this paper, are based on the assumption that $h(t)$ is band-limited such that only adjacent subcarrier channels overlap. As is discussed in Section 5, below, more recent developments on filter design for FBMC relax on this condition and assume overlapping can occur beyond adjacent bands as well. 


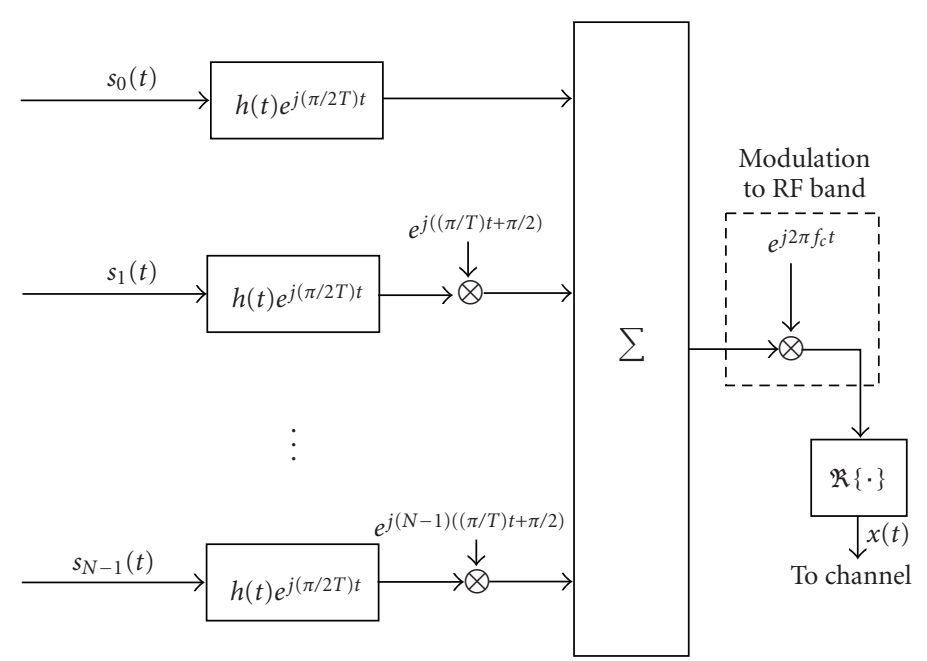

(a)

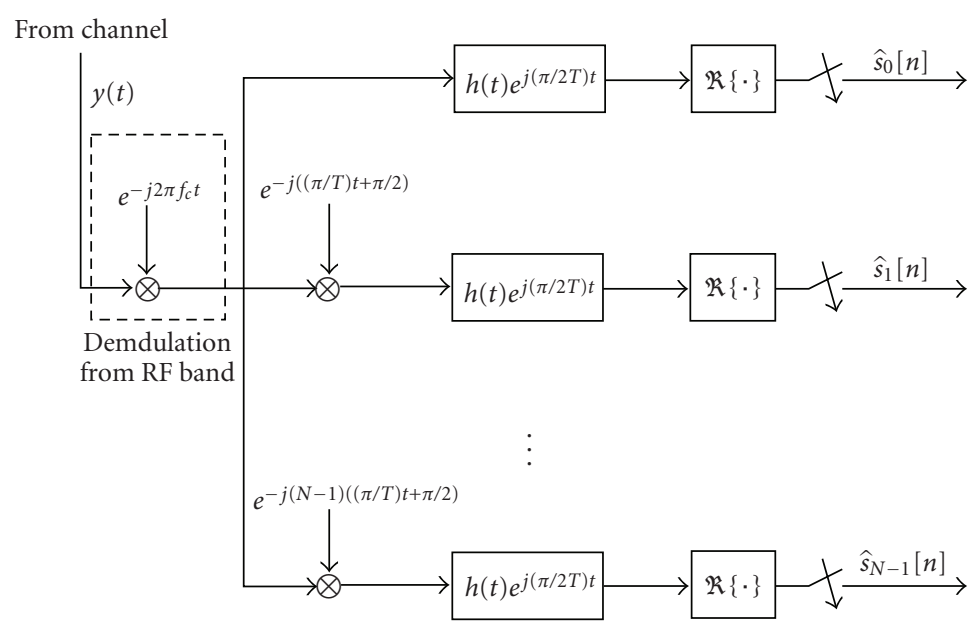

(b)

FIgURE 4: Block diagram of a CMT transceiver: (a) transmitter; (b) receiver.

The constraints mentioned in items (1) and (2) are standard results from the single carrier communication systems. Thus, we proceed with an attempt to clarify the reasoning behind the constraints mentioned in item (3). Figure 5(a) presents a pair of typical magnitude and phase responses of $H(f)$ with the additional phase symmetry condition mentioned in the constraints (3). Figure 5(b) presents the amplitude and phase responses of $H(f)$ after a right shift of $f=3 / 4 T$. This is the channel response corresponding to an adjacent subcarrier to the baseband channel, after demodulation at the receiver. Figure 5(c) presents the amplitude and phase responses of the VSB matched baseband filter at the receiver. Finally, Figure 5(d) shows the total channel response from an adjacent subcarrier to a demodulated subcarrier channel at the baseband. This has the transfer function $G_{1}(f)=j H^{*}(f-1 / 4 T) H(f-$ $3 / 4 T)$, where the factor $j$ arises from the phase shift of $\pi / 2$ among the adjacent subcarrier channels. As seen, as a consequence of the phase symmetry of $H(f)$ around $f=$ $1 / 4 T, G_{1}(f)$ has a phase response of $\pi / 2$. Moreover, the even symmetry of $|H(f)|$ leads to an even symmetric magnitude response $\left|G_{1}(f)\right|$ around the point $f=1 / 2 T$. Finally, taking the inverse Fourier transform of $G_{1}(f)$, at the time instant $t=n T$, one finds that $g_{1}(n T)$ is an imaginary number, for any integer value of $n$. Moreover, since in VSB demodulation after matched filtering the imaginary part of the result is ignored, this result shows that the combination of the phase symmetry of $H(f)$ around $f=1 / 4 T$ and the introduced phase shift of $\pi / 2$ between adjacent subcarrier channels lead to ICI cancellation.

\section{Staggered Modulated Multitone (SMT)}

Figure 6 presents the block diagram of an SMT transceiver. The data signals $s_{0}(t)$ through $s_{N-1}(t)$ are continuous-time signals associated with transmit symbol sequences that are defined as

$$
s_{k}(t)=\sum_{n=-\infty}^{\infty} s_{k}[n] \delta(t-n T), \quad \text { for } k=0,1, \ldots, N-1,
$$




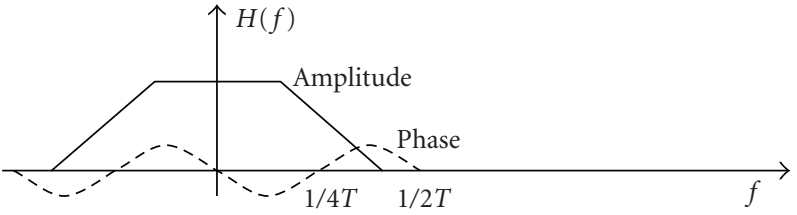

(a)

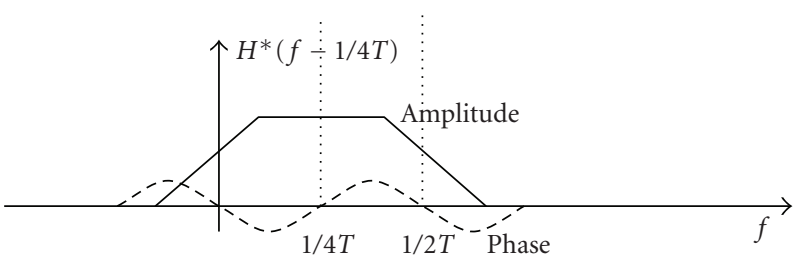

(c)

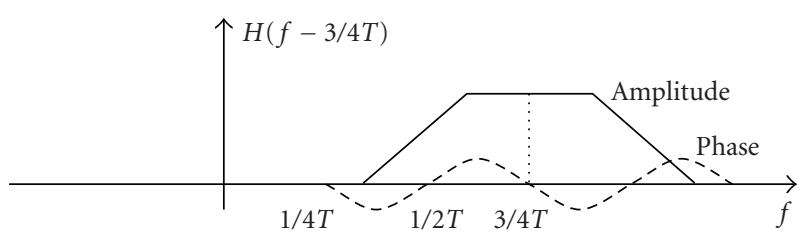

(b)

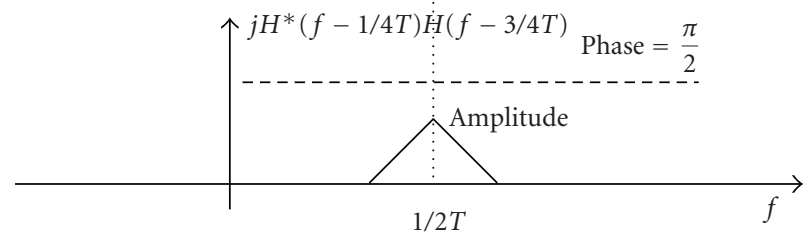

(d)

FIgURE 5: Demonstration of ICI cancellation in CMT when $H(f)$ has a relaxed phase response.

where $s_{k}[n]$ are complex-valued (e.g., QAM or PSK) data symbols that may be written as

$$
s_{k}[n]=s_{k}^{\mathrm{I}}[n]+j s_{k}^{\mathrm{Q}}[n],
$$

where the superscripts "I" and "Q" refer to the in-phase and quadrature parts, respectively. Note that at each subcarrier channel, the real and imaginary parts of $s_{k}[n]$ are separated and time staggered by $T / 2$. This is done through the pulse shaping filter $h(t)$ which is time shifted to the right on the quadrature branches. Also, note that the same filter $h(t)$ is used at both the transmitter and receiver sides. This, clearly, implies the symmetry condition $h(-t)=h(t)$ to guarantee a matched filter pair at the two sides. Moreover, we note that the multiplications by $1, e^{j((2 \pi / T) t+\pi / 2)}, \ldots, e^{j(N-1)((2 \pi / T) t+\pi / 2)}$ are effectively modulators that organize the subcarrier channels at the center frequencies $0,2 \pi / T, \ldots, 2 \pi(N-1) / T$. This means the spacing between the adjacent subcarriers is $1 / T$ which is twice of that of the CMT.

The detected data symbols at the receiver output are denoted as $\hat{s}_{k}[n]$. The filter $h(t)$ should be designed such that when channel is perfect (i.e., there is no multipath and/or no noise), $\hat{s}_{k}[n]=s_{k}[n]$. Next, we proceed and derive the condition that should be imposed on $h(t)$ for such a perfect recovery.

When only adjacent bands overlap and, thus, one can ignore possible interference from non-adjacent bands, interference may only happen in the following three different ways.

(1) Possible ISI across each phase or quadrature subcarrier channel, that is, the successive symbol values of $s_{k}^{\mathrm{I}}[n]$ may interfere with one another, and similarly for $s_{k}^{\mathrm{Q}}[n]$.

(2) Cross interference among the sequences $s_{k}^{\mathrm{I}}[n]$ and $s_{k}^{\mathrm{Q}}[n]$.

(3) ICI among the adjacent subcarrier signals.

To explore the interferences mentioned in items (1) and (2), we extract the relevant branches from Figure 6 that connects $s_{k}^{\mathrm{I}}(t)$ and $s_{k}^{\mathrm{Q}}(t)$ to $\hat{s}_{k}^{\mathrm{I}}[n]$ and $\hat{s}_{k}^{\mathrm{Q}}[n]$. In the absence of channel, these are presented in Figure 7(a). In presenting this figure, we have noted that the subcarrier modulator $e^{j k((2 \pi / T) t+\pi / 2)}$ and the demodulator $e^{-j k((2 \pi / T) t+\pi / 2)}$, and also the modulator to RF, $e^{j 2 \pi f_{c} t}$, and the demodulator from RF, $e^{-j 2 \pi f_{c} t}$, cancel each other. If we further note that the output of $h(t)$ on the top-left of Figure 7(a) is a real function of time, and the output of $h(t-T / 2)$ on the bottom-left of Figure 7(a) is an imaginary function of time, one can separate the blocks in Figure 7(a) in two separate channels as in Figure 7(b).

From Figure 7(b), we observe that there is no cross interference between the in-phase and quadrature of each subcarrier channel in SMT. To avoid ISI in the upper branch of Figure 7(b), it is necessary and sufficient that $h(t)$ be chosen such that the combined response $h(t) \star h(t)$ be a Nyquist pulse. This requirement also guarantees ISI free transmission in the lower (i.e., the quadrature) channel in Figure 7(b), since $h(t-T / 2) \star h(t+T / 2)=h(t) \star h(t)$ and this, in turn, implies that the lower channel also has a Nyquist response.

Figure 8 presents the relevant branches of the $k+1$ th subcarrier channel that may leak signal to the output of the $k$ th subcarrier channel of an SMT system. Note that, here, the outputs $\widehat{s}_{k}^{\mathrm{T}}[n]$ and $\widehat{s}_{k}^{\mathrm{Q}}[n]$ are replaced by $\widetilde{s}_{k}^{\mathrm{I}}[n]$ and $\widetilde{s}_{k}^{\mathrm{Q}}[n]$ to signify that they are interference terms. To explore the interference terms $\widetilde{s}_{k}^{T}[n]$ and $\widetilde{s}_{k}^{Q}[n]$, we study the impulse responses between each of the inputs and each of the outputs in Figure 8; a total of four impulse responses.

Let us begin with looking at the impulse response between the input $s_{k+1}^{\mathrm{I}}(t)$ and the output before the sampler in the upper-right branch of Figure 8. We obtain this by direct inspection of Figure 8 as

$$
\begin{aligned}
g_{1}(t) & =\Re\left\{h(t) e^{j((2 \pi / T t)+\pi / 2)}\right\} \star h(t) \\
& =-\int_{-\infty}^{\infty} h(\tau) \sin \left(\frac{2 \pi}{T} \tau\right) h(t-\tau) d \tau .
\end{aligned}
$$




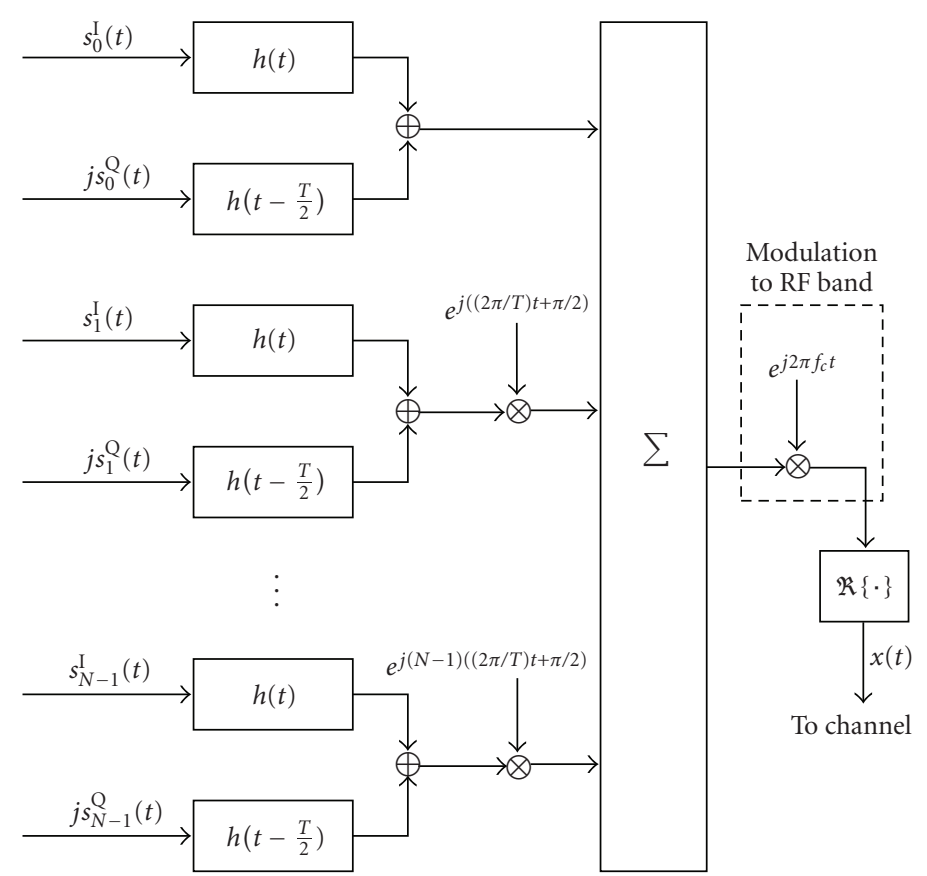

(a)

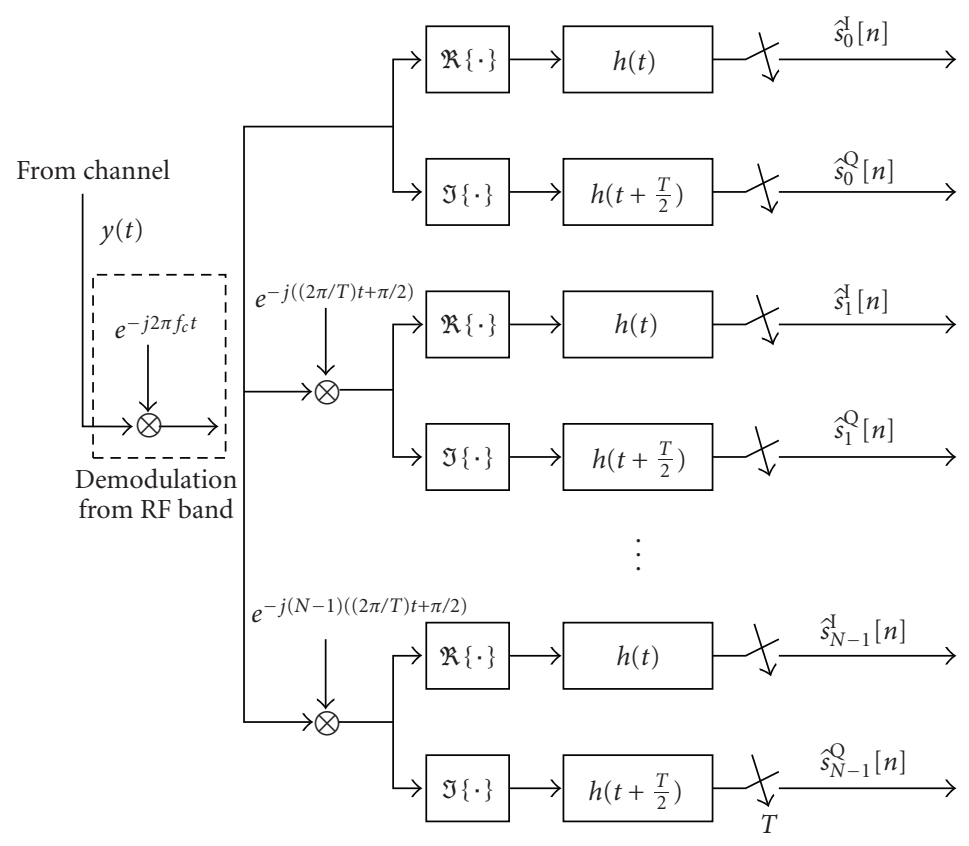

(b)

FIGURE 6: Block diagram of an SMT transceiver: (a) transmitter; (b) receiver.

Substituting $t=n T$ into (10), we obtain

$$
g_{1}(n T)=-\int_{-\infty}^{\infty} h(\tau) \sin \left(\frac{2 \pi}{T} \tau\right) h(n T-\tau) d \tau
$$

Applying the change of variable $\tau$ to $n T / 2+\tau$ to this result, we get

$$
\begin{aligned}
g_{1}(n T) & =(-1)^{n+1} \int_{-\infty}^{\infty} h\left(\frac{n T}{2}+\tau\right) h\left(\frac{n T}{2}-\tau\right) \sin \left(\frac{2 \pi}{T} \tau\right) d \tau \\
& =0 .
\end{aligned}
$$

This, clearly, implies that there is no interference from the symbol sequence $s_{k+1}^{\mathrm{I}}[n]$ to $\widehat{s}_{k}^{\mathrm{I}}[n]$. Following the same line of derivations, it is not difficult to show that the same is true for the rest of the paths in Figure 8. 


\section{Similarities and Differences of CMT and SMT}

From the derivations in Sections 2 and 3, one may find that the key point which results in ICI cancellation among adjacent subcarrier channels in both CMT and SMT is the fact that the same prototype filter $h(t)$ is used at both the transmitter and receiver sides; see (6) and (12) for similarities of the final results. It is interesting to note that ICI cancellation among adjacent subcarrier channels does not impose any other restriction on the choice of $h(t)$. The condition that $p(t)=h(t) \star h(t)$ be a Nyquist pulse, thus, $h(t)$ should be an even symmetric square-root Nyquist filter, is imposed to avoid ISI. Moreover, $h(t)$ was chosen to be band-limited to minimize ICI among non-adjacent subcarrier channels; a condition that will be relaxed in the next section. Also, as discussed in Section 2.3, for CMT, the even symmetry constraint of $h(t)$ can be relaxed, if the phase response of $H(f)$ satisfy an additional odd symmetry condition with respect to the midpoint of its transition band. It is straightforward to follow the same line of argument and show that the same is true in the case of SMT. Therefore, the fundamental concepts based on which both CMT and SMT have been developed are the same.

The main difference between CMT and SMT is the modulation type. In SMT, data symbols are QAM and, thus, the modulation is double side-band (DSB). In CMT, on the other hand, data symbols are PAM and, thus, in order to keep the same bandwidth efficiency, VSB modulation is used. Moreover, if we assume that each DSB subcarrier channel in SMT has the same width as a VSB subcarrier channel in CMT, one finds that symbol rate in each subcarrier channel of CMT will be double that of SMT. Next, we proceed to put these observations in a mathematical formulation.

From Figure 6, one finds the SMT signal before modulation to RF band is given by

$$
\begin{aligned}
v_{\mathrm{SMT}}(t)= & \sum_{l=0}^{N-1} \sum_{n=-\infty}^{\infty}\left(s_{l}^{\mathrm{I}}[n] h(t-n T)+j s_{l}^{\mathrm{Q}}[n] h\left(t-\frac{T}{2}-n T\right)\right) \\
& \times e^{j l((2 \pi / T) t+\pi / 2)} .
\end{aligned}
$$

On the other hand, from Figure 4, when $T$ is replaced by $T / 2$ (to equalize the subcarrier bandwidth of CMT with SMT), the CMT signal before modulation to RF band is obtained as

$$
\begin{aligned}
v_{\mathrm{CMT}}(t) & \\
= & \sum_{l=0}^{N-1} \sum_{n=-\infty}^{\infty} s_{l}[n] h\left(t-\frac{n T}{2}\right) e^{j(\pi / T)(t-n T / 2)} e^{j l((2 \pi / T) t+\pi / 2)} \\
= & \sum_{l=0}^{N-1} \sum_{n=-\infty}^{\infty}(-j)^{n} s_{l}[n] h\left(t-\frac{n T}{2}\right) e^{j(\pi / T) t} e^{j l((2 \pi / T) t+\pi / 2)} .
\end{aligned}
$$

It is instructive to note that both $v_{\mathrm{SMT}}(t)$ and $v_{\mathrm{CMT}}(t)$ are complex-valued baseband signals.
Separating the even and odd terms in (14), we obtain

$$
\begin{aligned}
v_{\mathrm{CMT}}(t)= & \sum_{l=0}^{N-1} \sum_{k=-\infty}^{\infty}(-j)^{2 k} s_{l}[2 k] h\left(t-\frac{(2 k) T}{2}\right) \\
& \times e^{j(\pi / T) t} e^{j l((2 \pi / T) t+\pi / 2)} \\
& +\sum_{l=0}^{N-1} \sum_{k=-\infty}^{\infty}(-j)^{2 k+1} s_{l}[2 k+1] h\left(t-\frac{(2 k+1) T}{2}\right) \\
& \times e^{j(\pi / T) t} e^{j l((2 \pi / T) t+\pi / 2)} \\
= & \sum_{l=0}^{N-1} \sum_{k=-\infty}^{\infty}(-1)^{k} s_{l}[2 k] h(t-k T) e^{j(\pi / T) t} e^{j l((2 \pi / T) t+\pi / 2)} \\
& +\sum_{l=0}^{N-1} \sum_{k=-\infty}^{\infty} j(-1)^{k+1} s_{l}[2 k+1] h\left(t-\frac{T}{2}-k T\right) \\
& \times e^{j(\pi / T) t} e^{j l((2 \pi / T) t+\pi / 2)} \\
= & \sum_{l=0}^{N-1} \sum_{k=-\infty}^{\infty}\left((-1)^{k} s_{l}[2 k] h(t-k T)\right. \\
& \times e^{j(\pi / T) t} e^{j l((2 \pi / T) t+\pi / 2)} .
\end{aligned}
$$

Now, if we remap the bits such that $s_{l}^{\mathrm{I}}[n]=(-1)^{k} s_{l}[2 k]$ and $s_{l}^{\mathrm{Q}}[n]=(-1)^{k+1} s_{l}[2 k+1]$, we find that

$$
v_{\mathrm{CMT}}(t)=v_{\mathrm{SMT}}(t) e^{j(\pi / T) t} .
$$

Applying Fourier transform to both sides of (16), we obtain

$$
V_{\mathrm{CMT}}(f)=V_{\mathrm{SMT}}\left(f-\frac{1}{4 T}\right) .
$$

These results show that there is a simple relationship between CMT and SMT. The complex-valued baseband signal $v_{\mathrm{CMT}}(t)$ can be constructed by first synthesizing the corresponding $v_{\mathrm{SMT}}(t)$ signal and then modulating the results with the complex-valued sine-wave $e^{j(\pi / T) t}$. Alternatively, one may start with synthesizing a respective $v_{\mathrm{CMT}}(t)$ signal and modulate the result with $e^{-j(\pi / T) t}$ to obtain a desired $v_{\mathrm{SMT}}(t)$ baseband signal. These also apply to the respective analysis filter banks. This observation has the following implications.

(i) SMT and CMT are equally sensitive to channel impairments, including time and frequency spread, carrier frequency offset and timing offset. Therefore any analysis done for one is applicable to the other.

(ii) A few structures have been proposed for efficient implementation of SMT (often referred to as OFDMOQAM), [18, 19]. These structures, with minor modifications, are readily applicable to CMT. 


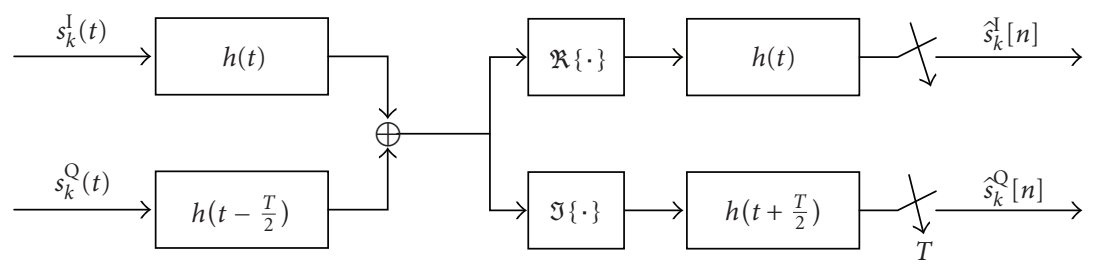

(a)
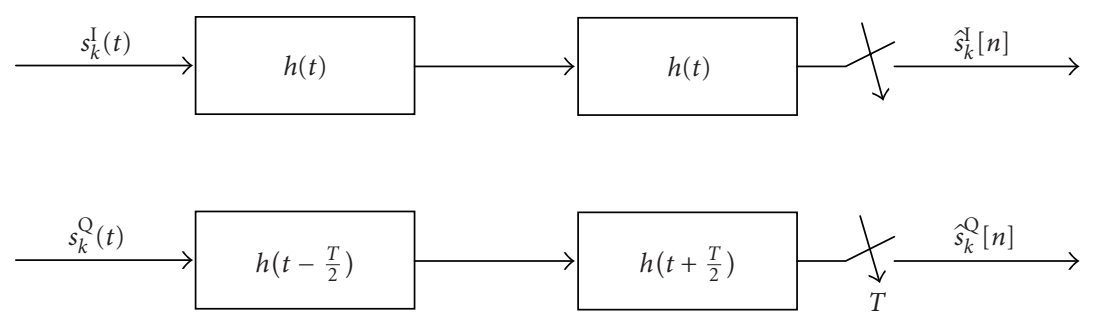

(b)

FIGURE 7: The $k$ th subcarrier channel in an SMT system.

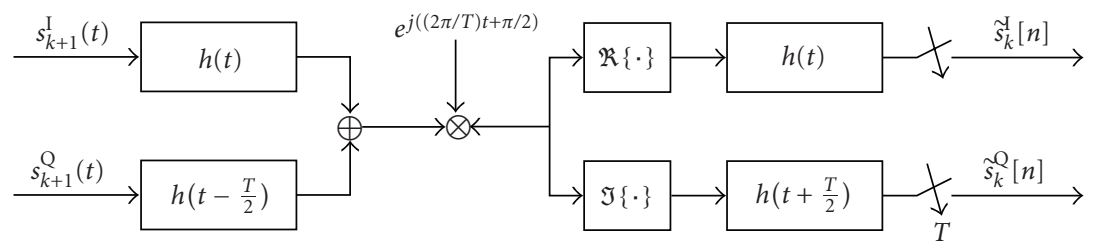

FIGURE 8: The $k$ th subcarrier channel in an SMT system.

A detailed study that evaluates sensitivity of CMT and SMT to channel impairments, through independent theoretical derivations for both methods, is presented in [56]. The results of this analysis formally confirm the accuracy of the first statement.

\section{Doubly Spread Channels and Prototype Filter Design}

The FBMC approaches proposed by Chang [7] and Saltzberg [8], and the polyphase implementation and equalization structures developed by Hirosaki [12] emphasize on channels with spreading in the time domain only. Possible channel variation in time that leads to spreading in the frequency domain was ignored. Later developments, starting with the pioneering work of Le Floch et al. [42], noted that in some wireless channels both time and frequency spread may be equally important and thus proposed modifications to the SMT prototype filters to limit them equally in time and frequency domains. These may be referred to as designs for doubly dispersive/spread channels.

Part of this section is devoted to a tutorial presentation of prototype filters that are designed for doubly spread channels. However, we note that although such filters are near optimal in time-frequency localization, that is, they attempt to equally limit the filter length in both the time and frequency domain, they are not necessarily the best designs for an arbitrary time-varying channel scenario. For instance, if a channel variation is very slow, but its time spread is significant, a design that emphasizes on confining the filter response within a minimum bandwidth by using a filter whose impulse response may spread over a long period of time can be a much better choice (of course, ignoring other factors such as complexity and transmission delay). Also, assuming that the channel statistics are known, the optimal design presented in [43] is not the one that equalizes the time and frequency spread of the prototype filter. Nevertheless, the solutions that equally weigh time and frequency spread are widely accepted as they provide good compromised designs.

5.1. Time-Frequency Localized Prototype Filters. Given a time-symmetric signal $s(t)$ and its Fourier transform $S(f)$, we define the time and frequency standard deviations

$$
\begin{gathered}
\sigma_{t}=\sqrt{\int_{-\infty}^{\infty} t^{2}|s(t)|^{2} d t}, \\
\sigma_{f}=\sqrt{\int_{-\infty}^{\infty} f^{2}|S(f)|^{2} d f .}
\end{gathered}
$$

The Heisenberg-Gabor uncertainty principle states that [57]

$$
\sigma_{t} \sigma_{f} \geq \frac{1}{4 \pi}
$$

In (19), the equality holds when $s(t)$ is the Gaussian pulse

$$
s(t)=e^{-\pi t^{2}} .
$$


Also, the Gaussian pulse $s(t)$ has the interesting property that $S(f)=s(f)$, that is, it is the same function in both time and frequency domains. Thus, any deviation from the Gaussian pulse, say, to reduce $\sigma_{t}$, that is, spreading of the signal in the time domain, will result in an increase of $\sigma_{f}$, that is, spreading in the frequency domain. Therefore, the Gaussian pulse (20) is optimal in the sense that it minimizes the time-frequency product $\sigma_{t} \sigma_{f}$ and also satisfies the desirable property of equal spreading in both time and frequency domain. However, unfortunately, the Gaussian pulse does not satisfy the Nyquist and other properties of $h(t)$ which were stated in Sections 2 and 3 for ISI and ICI cancellation in CMT and SMT systems. Noting this, Le Floch et al. [42] and Haas and Belfiore [44] have proposed two different methods for designing pulse shapes that satisfy the conditions necessary for ISI and ICI cancellation in CMT and SMT and result in a time-frequency product that is only slightly greater than the lower limit given by (19).

Both design methods in $[42,44]$ are developed using the time-frequency ambiguity function

$$
A(\tau, f)=\int_{-\infty}^{\infty} h(t) h(t+\tau) e^{-j 2 \pi f t} d t .
$$

The ambiguity function $A(\tau, f)$ has the following interpretation. For $f=0$,

$$
A(\tau, 0)=\int_{-\infty}^{\infty} h(t) h(t+\tau) d t=h(\tau) \star h(-\tau)
$$

and the constraints $A(n T, 0)=0$, for $n \neq 0$, imply that $h(t)$ is a square-root Nyquist filter, hence, a sequence of data symbols that are $T$ spaced can be received free of ISI. On the other hand, the constraints $A(0, k \Delta f)=0$, for any $k$, imply that a pair of modulated filters that are spaced across frequency by $k \Delta f$ do not introduce ICI on each other. Accordingly, if an FBMC system is constructed based on a prototype filter $h(t)$ whose ambiguity function satisfies the constraints

$$
A(n T, k \Delta f)=0, \quad \text { for } n \neq 0, \text { and any } k,
$$

where $T$ is the symbol spacing and $\Delta f$ is carrier spacing, in the absence of channel distortion, ISI and ICI free transmission is achieved.

It has been noted that to achieve a reasonable timefrequency localization which results in a value of $\sigma_{t} \sigma_{f}$ close to the lower limit of $1 / 4 \pi, T \Delta f$ should be given a value greater than $1[42,44]$. On the other hand, $T \Delta f=2$ turns out to be a good choice as it results in prototype filters with a value of $\sigma_{t} \sigma_{f}$ close to the lower limit $1 / 4 \pi$ and also, as discussed below, is the choice that leads to CMT and SMT systems.

When an FBMC system with a prototype filter that satisfies the constraints (23) as well as the equality $T \Delta f=2$ is implemented, complex-valued (i.e., QAM or PSK) data symbols that are spread over a grid of points in the timefrequency phase space at locations $n T$ and $2 k / T$ can be transmitted free of ISI and ICI (assuming an ideal channel) $[42,44]$. This grid of points are shown in Figure 9, marked as -. One may also note that this grid of points have a density of $1 /(T \Delta f)=0.5$.

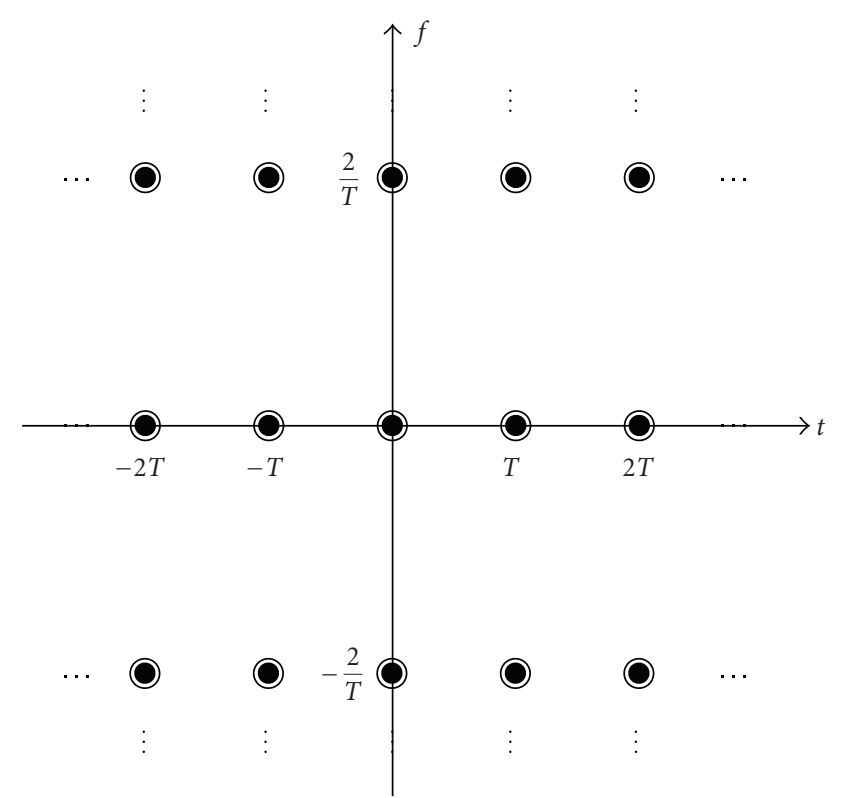

FIGURE 9: Time-frequency phase space for transmission in an FBMC system with QAM symbols.

The trick in SMT and CMT is that replacing complexvalued symbols by real-valued (PAM) symbols, it is possible to double the density of the grid points both along the time and frequency axes. This increases the density of the grid points by a factor of 4 . However, since each real symbol is equivalent to half of a complex symbol, this leads to an effective density of one complex symbol per unit area. The principle behind ISI and ICI cancellation after adding the intermediate points lies in the introduction of $\pi / 2$ phase rotations and the fact that only real or imaginary parts of the analyzed signals are preserved at the receiver outputs. The mathematical derivations that were presented in Sections 2 and 3, for the case where only adjacent channels overlap, can be easily extended to any CMT or SMT system whose prototype filter satisfies the constraints (23).

Figure 10 presents a grid of points in a phase space for the case of SMT. The points where an even and odd factors of $\pi / 2$ phase are applied to the respective symbols are indicated as and $\mathrm{O}$, respectively. A grid of points that corresponds to a CMT system is presented in Figure 11. As one would expect, we note that the density of the grid points in both Figures 10 and 11 are the same. This implies that, as discussed before, SMT and CMT have the same spectral efficiency. Moreover, one may note that the time-frequency phase space shown in Figure 11 is obtained from the one in Figure 10 after stretching the time axis by a factor of 2, compressing the frequency axis by a factor of 0.5 , and moving the grid points upward by one half of symbol spacing. This, clearly, is another interpretation of the relationship between CMT and SMT that was developed in Section 4.

Next, we proceed with a brief discussion of three common types of prototype filters that have appeared in the literature. 


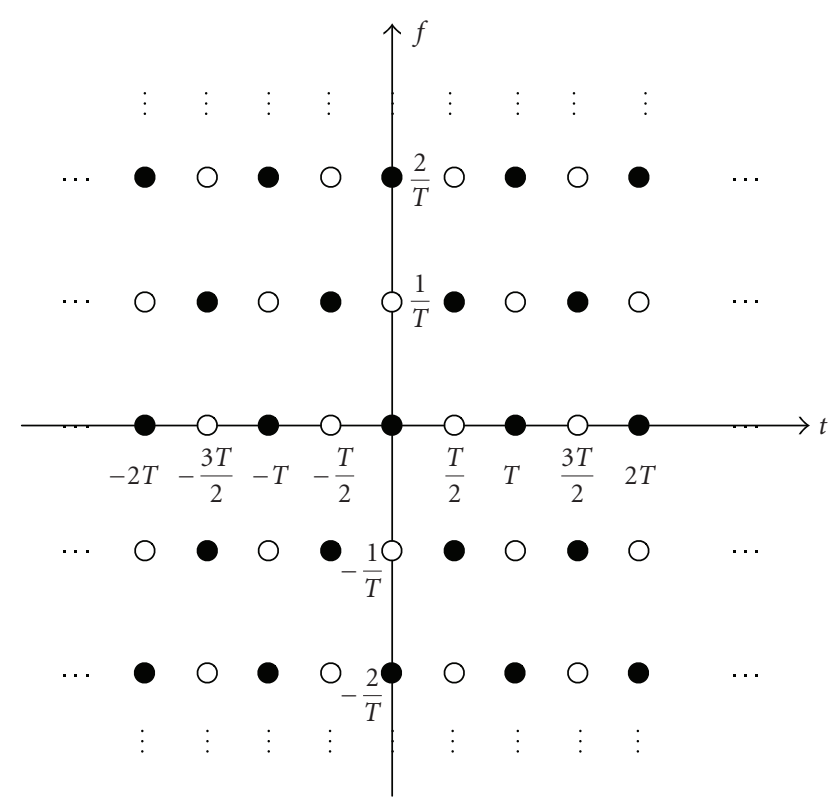

FIgure 10: Time-frequency phase space for transmission in SMT.

5.2. Prototype Filter Design for Time-Invariant Channels. When the channel is a frequency-selective time-invariant one, a fair criterion for designing the prototype filter is minimization of its bandwidth. This results in minimum variation of the channel gain across each subcarrier band, and thus an approximation of flat fading for each subcarrier channel becomes more acceptable. Hence, the use of a single complex-tap equalizer per subcarrier becomes more acceptable. If no constraint is applied to the desired prototype filter, the optimum design will be an ideal filter with the transfer function

$$
H(f)= \begin{cases}1, & |f| \leq \frac{1}{2 T} \\ 0, & \text { otherwise. }\end{cases}
$$

This is a square-root Nyquist filter with roll-off factor $\alpha=0$, thus, results in an infinite length and, hence, an unrealizable, filter. To get a realizable filter, a roll-off factor $\alpha>0$ is introduced. In particular, any square-root raised-cosine filter with a roll-off factor $0<\alpha \leq 1$ will result in a realizable SMT system with perfect ISI and ICI cancellation. In the pioneering work [8] and also in [42], the choice of

$$
H(f)= \begin{cases}\cos \left(\frac{\pi f T}{2}\right), & |f| \leq \frac{1}{T} \\ 0, & \text { otherwise }\end{cases}
$$

which is a square-root raised-cosine filter with roll-off factor $\alpha=1$ was suggested. This selection of $H(f)$ provides a good compromise solution. Because of its relatively relaxed transition bands, it can be well approximated with a relatively short filter, and still can achieve a very high attenuation in the stopband. Design methods that find optimum filters with finite length and good attenuation in the stopband have been reported in the literature $[55,58]$.

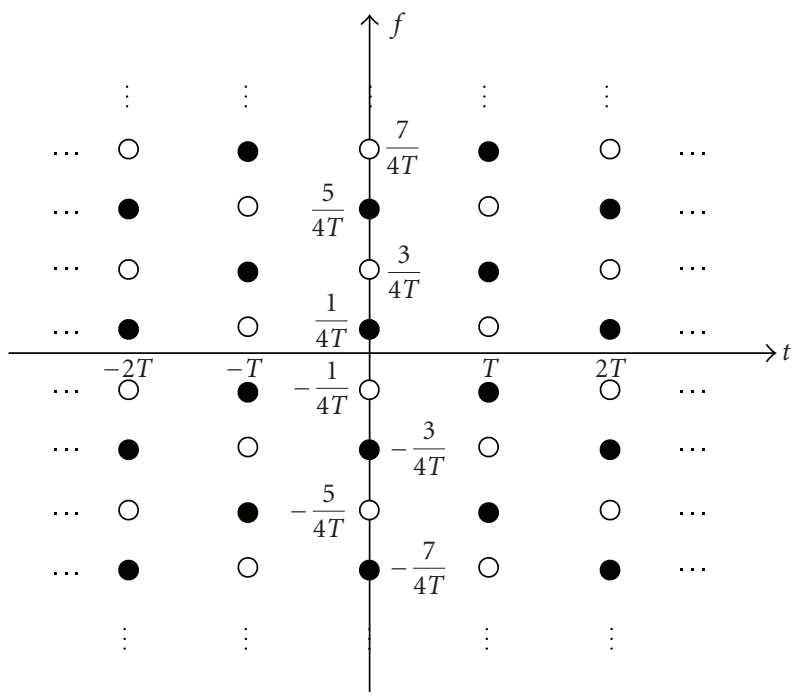

FIgURE 11: Time-frequency phase space for transmission in CMT.

5.3. Isotropic Orthogonal Transform Algorithm (IOTA) Prototype Filter Design. IOTA design/algorithm was first introduced by Alard [59] and was put in archival journals by Le Floch et al. [42]; see also [60] for further developments. The algorithm starts with the Gaussian pulse $g_{\alpha}(t)=\sqrt[4]{2 \alpha} e^{-\pi \alpha t^{2}}$ and convert it to the orthogonalized pulse

$$
h_{\alpha}(t)=\mathcal{O}_{\tau_{0}} \mathcal{F}^{-1} \mathcal{O}_{v_{0}} \mathcal{F} g_{\alpha}(t),
$$

where the parameters $\tau_{0}$ and $\nu_{0}$ are defined below, $\mathcal{F}$ and $\mathcal{F}^{-1}$, respectively, denote Fourier and inverse Fourier transforms, and $\mathcal{O}_{a}$ is an orthogonalization operator defined as

$$
y(u)=\frac{x(u)}{\sqrt{a \sum_{k=-\infty}^{\infty}|x(u-k a)|^{2}}} .
$$

This procedure results in a pulse shape $h_{\alpha}(t)$ which after applying time scaling to it can be converted to a filter that satisfies the ambiguity function constraints given in (23); see $[42,60]$ for more details.

5.4. Hermite Functions Based Prototype Filter Design. Haas and Belfiore [44] noted that the set of functions

$$
D_{n}(t)=h_{n}(\sqrt{2 \pi} t), \quad \text { for } n=0,4,8, \ldots
$$

where $h_{n}(t)=e^{-t^{2} / 2}\left(d^{n} / d t^{n}\right) e^{-t^{2}}$, satisfy the identity $\mathcal{F} D_{n}(t)=D_{n}(f)$. They have thus concluded that a pulse shape $h(t)$ formed by linearly combining $D_{n}(t)$, for $n=$ $0,4,8, \ldots$ also satisfies the identity $\mathcal{F} h(t)=h(f)$. They have then presented a procedure for combining $D_{n}(t)$ functions to construct a pulse shape $h(t)$ whose ambiguity function satisfies the constraints (23).

5.5. Numerical Results and Comparisons. To develop more insight to the differences of the various prototype filter designs, in Figures 12 and 13, respectively, the time and 


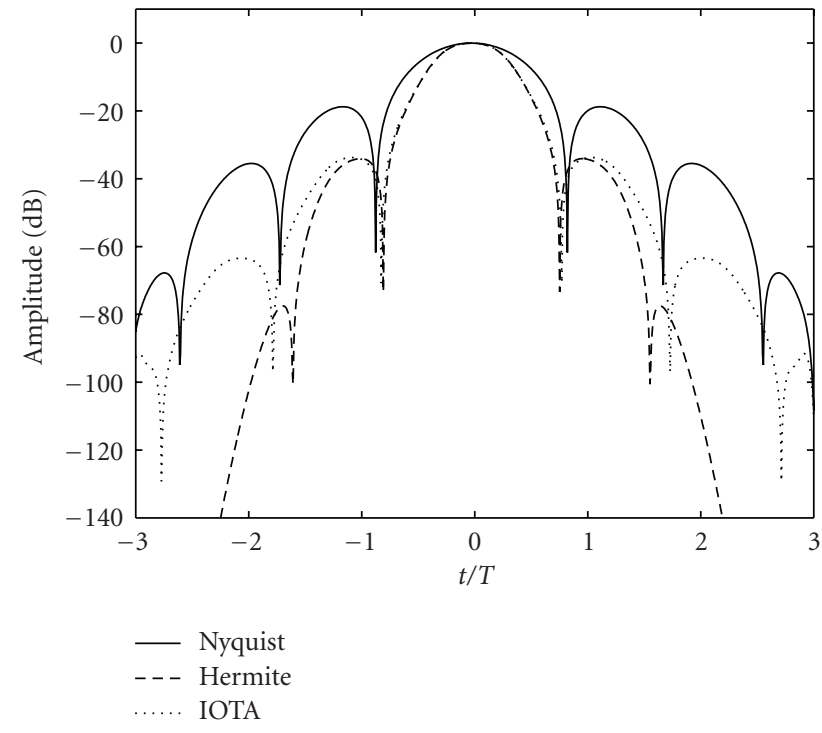

FIgURE 12: Magnitude of impulse response of three designs of prototype filter of length $6 \mathrm{~T}$.

frequency domain responses of a Nyquist design (with rolloff factor of 1) [58], a Hermite design [44], and an IOTA design $[42,60]$ are presented. All filters are designed for a finite duration time response $6 \mathrm{~T}$. As one would expect, the Nyquist design provides the narrowest response in the frequency domain, at a cost of a wider response in the time domain. IOTA and Hermite designs, for most parts, are quite similar. Hermite design outperforms IOTA design over the intervals of time and frequency that the magnitude of the responses are below $-40 \mathrm{~dB}$. We may also note that while the pass and transition bands of the Nyquist design is over the interval $-1 / T$ to $1 / T$, this is much wider in the cases of Hermite and IOTA designs.

\section{Channel Impact and Equalization}

The derivations and discussions so far were based on the assumption that the channel was ideal, that is, a channel with a constant gain and a constant group delay across the frequency band that includes all the subcarrier channels. However, we note that the main reason for using any multicarrier technique, including CMT and SMT, is to deal with frequency selective channels, that is, the channels whose gain vary across the frequency band and thus may suffer from a significant level of ISI and ICI. On the other hand, the most important advantage of multicarrier techniques is that they greatly simplify the task of channel equalization a mechanism that is used to combat ISI and ICI. In a single carrier system, when the channel suffers from a significant level of ISI, a transveral/FIR filter with many taps have to be used to generate a response that resembles the inverse of the channel gain across the transmission band. Such inversion will result in noise enhancement across the portion of the frequency band that the channel gain is low [61]. Adaptation of the equalizer tap weights also may not be a straightforward task. Wireless multipath channels are always time-varying

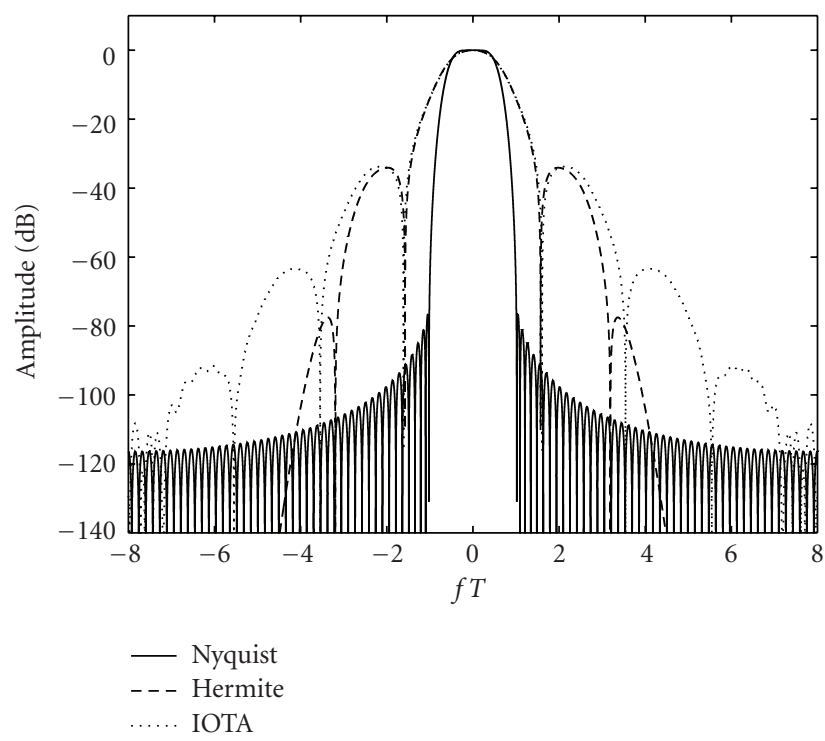

Figure 13: Magnitude of frequency response of three designs of prototype filter of length $6 T$.

and thus the equalizer should be adapted to track channel variations. The speed of tracking decreases as the length of equalizer increases $[62,63]$. Hence, when the channel is highly frequency selective and as a result a long equalizer has to be used, an equalizer adaptation algorithm may not be able to cope with the channel variation.

The above problems are solved or, at least, greatly moderated when a multicarrier method is adopted. In the case of OFDM, as long as the duration of the channel impulse response is shorter than the cyclic prefix length and channel variation over each OFDM symbol is negligible, a frequency selective channel converts to a number of subcarrier channels with flat gains. In CMT and SMT, the assumption of a flat gain over each subcarrier channel is true only approximately. However, the accuracy of this approximation improves as the bandwidth of each subcarrier channel decreases. Here, the bandwidth is defined as a frequency range that include the pass and transition bands of each subcarrier channel. Like OFDM, in FBMC systems also equalization is performed separately on each subcarrier channel. In [24] and many subsequent papers on DWMT, for example [2629], subcarrier equalization was performed by combining signals from a center and two adjacent subcarrier bands. This which results in an equalizer with many taps per subcarrier, was later proved to be unnecessary and a much simpler equalizer that effectively needs two real taps per subcarrier is sufficient [30]. The pioneering work of Hirosaki [12], that explored polyphase structures for SMT, has introduced an equalization technique similar to [30]. However, this early work apparently remained unnoticed to the researchers on DWMT/CMT, probably because they saw DWMT/CMT as a method significantly different from SMT.

Horosaki [12] also explored the case where each subcarrier band within an SMT system could not be approximated by a flat gain. He showed, in such cases, to preserve the orthogonality of each subcarrier channel with its adjacent 
bands, the equalizer at each subcarrier channel should be a fractionally spaced one. The sampling at each subcarrier channel should be equal to the total bandwidth of the respective subcarrier signal. This in the case of the Nyquist prototype filter that was presented in the previous section results in a $T / 2$-spaced equalizer. In the cases of IOTA and Hermite filters equalizers with more closely spaced taps are required since these filters have wider bandwidths than their Nyquist counterpart.

It is also worth noting that equalization is one of the least explored issues in FBMC systems and thus further research in this is necessary. In doubly spread channels, in particular, the use of IOTA and/or Hermite prototype filters is intuitively sound. However, this is without consideration of the practical fact that adaptive equalizers with nontrivial tracking algorithms may change the balance between a good prototype filter and an increase in the number of equalizer taps for a given performance-good filters like IOTA and Hermite may need the use of fractionally spaced equalizer with more taps than a Nyquist based system and thus may suffer more from slow convergence and/or tracking problems.

\section{Conclusion}

A tutorial overview of two filter bank multicarrier (FBMC) techniques that were proposed in the early days of development of digital communication systems was presented. The first method constructs a multicarrier signal by aggregating a number of vestigial side band (VSB) signals that carry a set of pulse amplitude modulated (PAM) symbol sequences. The second method, on the other hand, transmits a set of staggered quadrature amplitude modulated (QAM) symbol sequences. Both methods achieve maximum bandwidth efficiency by using subbcarrier signals that are minimally spaced and designed such that could be perfectly separated at the receiver side. It was also shown that these two methods are closely related through a modulation step and a oneto-one mapping of data symbols. Additional advancements in further development of FBMC systems, particularly, the various approaches that have been proposed for designing the underlying prototype filters were also reviewed and compared against each other.

\section{Acknowledgments}

The authors are grateful to anonymous reviewers whose constructive comments led to a significant improvement of this paper. They are also grateful to Pierre Siohan for providing them the MATLAB code for generating the IOTA filters. This work was supported by the National Science Foundation Award 0801641.

\section{References}

[1] R. Van Nee and R. Prasad, OFDM for Wireless Multimedia Communications, Arthec House, Boston, Mass, USA, 2000.

[2] Y. Li and G. L. Stüber, Eds., Orthogonal Frequency Division Multiplexing for Wireless Communications, Springer, New York, NY, USA, 2006.
[3] "Air interface for fixed and mobile broadband wireless access systems," IEEE Std. 802.16e, 2005.

[4] T. A. Weiss and F. K. Jondral, "Spectrum pooling: an innovative strategy for the enhancement of spectrum efficiency," IEEE Communications Magazine, vol. 42, no. 3, pp. S8-S14, 2004.

[5] M. Morelli, C.-C. Jay Kuo, and M.-O. Pun, "Synchronization techniques for orthogonal frequency division multiple access (OFDMA): a tutorial review," Proceedings of IEEE, vol. 95, no. 7, pp. 1394-1427, 2007.

[6] B. Farhang-Boroujeny and R. Kempter, "Multicarrier communication techniques for spectrum sensing and communication in cognitive radios," IEEE Communications Magazine, vol. 46, no. 4, pp. 80-85, 2008.

[7] R. W. Chang, "High-speed multichannel data transmission with bandlimited orthogonal signals," The Bell System Technical Journal, vol. 45, pp. 1775-1796, 1966.

[8] B. R. Saltzberg, "Performance of an efficient parallel data transmission system," IEEE Transactions on Communications Technology, vol. 15, no. 6, pp. 805-811, 1967.

[9] M. Bellanger and J. Daguet, "TDM-FDM transmultiplexer: digital polyphase and FFT," IEEE Transactions on Communications, vol. 22, no. 9, pp. 1199-1205, 1974.

[10] G. Bonnerot, M. Coudreuse, and M. Bellanger, "Digital processing techniques in the 60 channel transmultiplexer," IEEE Transactions on Communications, vol. 26, no. 5, pp. 698706, 1978.

[11] B. Hirosaki, "An analysis of automatic equalizers for orthogonally multiplexed QAM systems," IEEE Transactions on Communications Systems, vol. 28, no. 1, pp. 73-83, 1980.

[12] B. Hirosaki, "An orthogonally multiplexed QAM system using the discrete fourier transform," IEEE Transactions on Communications Systems, vol. 29, no. 7, pp. 982-989, 1981.

[13] H. Bölcskei, P. Duhamel, and R. Hleiss, "Orthogonalization of OFDM/OQAM pulse shaping filters using the discrete Zak transform," Signal Processing, vol. 83, no. 7, pp. 1379-1391, 2003.

[14] H. Bölcskei, "Orthogonal frequency division multiplexing based on offset QAM," in Advances in Gabor Analysis, H. G. Feichtinger and T. Strohmer, Eds., Birkhäuser, Boston, Mass, USA, 2003.

[15] H. Bölcskei, P. Duhamel, and R. Hleiss, "Design of pulse shaping OFDM-OQAM systems for high data-rate transmission over wireless channels," in Proceedings of the IEEE International Conference on Communications (ICC '99), vol. 1, pp. 559-564, Vancouver, Canada, June 1999.

[16] B. Hirosaki, S. Hasegawa, and A. Sabato, "Advanced groupband data modem using orthogonally multiplexed QAM technique," IEEE Transactions on Communications, vol. 34, no. 6, pp. 587-592, 1986.

[17] H. H. Chen and X. D. Cai, "Waveform optimization for OQAM-OFDM systems by using nonlinear programming algorithms," in Proceedings of the 47th IEEE Vehicular Technology Conference (VTC'97), pp. 1385-1389, Phoenix, Ariz, USA, May 1997.

[18] G. Cariolaro and F. C. Vagliani, "An OFDM scheme with a half complexity," IEEE Journal on Selected Areas in Communications, vol. 13, no. 9, pp. 1586-1599, 1995.

[19] L. Vangelista and N. Laurenti, "Efficient implementations and alternative architectures for OFDM-OQAM systems," IEEE Transactions on Communications, vol. 49, no. 4, pp. 664-675, 2001. 
[20] S. Pfletschinger and J. Speidel, "Optimized impulses for multicarrier offset-QAM," in Proceedings of the IEEE Global Telecommunications Conference (Globecom '01), pp. 207-211, San Antonio, Texas, USA, November 2001.

[21] P. Siohan, C. Siclet, and N. Lacaille, "Analysis and design of OFDM/OQAM systems based on filterbank theory," IEEE Transactions on Signal Processing, vol. 50, no. 5, pp. 1170-1183, 2002.

[22] A. Viholainen, T. Saramäki, and M. Renfors, "Cosinemodulated filter bank design for multicarrier VDSL modems," in Proceedings of the 6th IEEE International Workshop on Intelligent SP and Communications Systems, pp. 143-147, Melbourne, Australia, November 1998.

[23] M. A. Tzannes, M. C. Tzannes, and H. Resnikoff, "The DWMT: a multicarrier transceiver for ADSL using M-band wavelet transforms," Standards Project ANSI Contribution T1E1.4/93-067, 1993.

[24] M. A. Tzannes, M. C. Tzannes, J. Proakis, and P. N. Heller, "DMT systems, DWMT systems and digital filter banks," in Proceedings of the IEEE International Conference on Communications (SUPERCOMM/ICC '94), vol. 1, pp. 311-315, New Orleans, La, USA, May 1994.

[25] S. D. Sandberg and M. A. Tzannes, "Overlapped discrete multitone modulation for high speed copper wire communications," IEEE Journal on Selected Areas in Communications, vol. 13, no. 9, pp. 1571-1585, 1995.

[26] A. D. Rizos, J. G. Proakis, and T. Q. Nguyen, "Comparison of DFT and cosine modulated filter banks in multicarrier modulation," in Proceedings of the IEEE Global Telecommunications Conference (Globecom '01), vol. 2, pp. 687-691, San Francisco, Calif, USA, November 1994.

[27] M. Hawryluck, A. Yongacoglu, and M. Kavehrad, "Efficient equalization of discrete wavelet multitone over twisted pair," in Proceedings of the International Zurich Seminar on Broadband Communications, pp. 185-191, Zurich, Switzerland, 1998.

[28] S. Govardhanagiri, T. Karp, P. Heller, and T. Nguyen, "Performance analysis of multicarrier modulation systems using cosine modulated filter banks," in Proceedings of the IEEE International Conference on Acoustics, Speech and Signal Processing (ICASSP '99), vol. 3, pp. 1405-1408, Phoenix, Ariz, USA, March 1999.

[29] B. Farhang-Boroujeny and W. H. Chin, "Time domain equaliser design for DWMT multicarrier transceivers," Electronics Letters, vol. 36, no. 18, pp. 1590-1592, 2000.

[30] B. Farhang-Boroujeny, "Multicarrier modulation with blind detection capability using cosine modulated filter banks," IEEE Transactions on Communications, vol. 51, no. 12, pp. 20572070, 2003.

[31] P. P. Vaidyanathan, Multirate Systems and Filter Banks, Prentice Hall, Englewood Cliffs, NJ, USA, 1993.

[32] S. Galli and O. Logvinov, "Recent developments in the standardization of power line communications within the IEEE," IEEE Communications Magazine, vol. 46, no. 7, pp. 6471, 2008.

[33] N. J. Fliege, Multirate Digital Signal Processing, John Wiley \& Son, New York, NY, USA, 1994.

[34] N. J. Fliege, "Modified DFT polyphase SBC filter banks with almost perfect reconstruction," in Proceedings of the International Conference on Acoustics, Speech, and Signal Processing (ICASSP '94), vol. 3, pp. 149-152, Adelaide, Australia, April 1994.
[35] T. Karp and N. J. Fliege, "Modified DFT filter banks with perfect reconstruction," IEEE Transactions on Circuits and Systems II: Analog and Digital Signal Processing, vol. 46, no. 11, pp. 1404-1414, 1999.

[36] R. Bregović and T. Saramäki, "A systematic technique for designing linear-phase FIR prototype filters for perfectreconstruction cosine-modulated and modified DFT filterbanks," IEEE Transactions on Signal Processing, vol. 53, no. 8, pp. 3193-3201, 2005.

[37] S. Salcedo-Sanz, F. Cruz-Roldan, and X. Yao, "Evolutionary design of digital filters with application to subband coding and data transmission," IEEE Transactions on Signal Processing, vol. 55, no. 4, pp. 1193-1203, 2007.

[38] P. N . Heller, T. Karp, and T. Q. Nguyen, "A general formulation of modulated filter banks," IEEE Transactions on Signal Processing, vol. 47, no. 4, pp. 986-1002, 1999.

[39] R. D. Gitlin and E. Y. Ho, “The performance of staggered quadrature amplitude modulation in the presence of phase jitter," IEEE Transactions on Communications, vol. 23, no. 3, pp. 348-352, 1975.

[40] H. Bölcskei, "Blind estimation of symbol timing and carrier frequency offset in wireless OFDM systems," IEEE Transactions on Communications, vol. 49, no. 6, pp. 988-999, 2001.

[41] H. Bölcskei, P. Duhamel, and R. Hleiss, "A subspace-based approach to blind channel identification in pulse shaping OFDM/OQAM systems," IEEE Transactions on Signal Processing, vol. 49, no. 7, pp. 1594-1598, 2001.

[42] B. Le Floch, M. Alard, and C. Berrou, "Coded orthogonal frequency division multiplex," Proceedings of the IEEE, vol. 83, no. 6, pp. 982-996, 1995.

[43] W. Kozek and A. F. Molisch, "Nonorthogonal pulseshapes for multicarrier communications in doubly dispersive channels," IEEE Journal on Selected Areas in Communications, vol. 16, no. 8, pp. 1579-1589, 1998.

[44] R. Haas and J.-C. Belfiore, "A time-frequency well-localized pulse for multiple carrier transmission," Wireless Personal Communications, vol. 5, no. 1, pp. 1-18, 1997.

[45] T. Hunziker and D. Dahlhaus, "Iterative detection for multicarrier transmission employing time-frequency concentrated pulses," IEEE Transactions on Communications, vol. 51, no. 4, pp. 641-651, 2003.

[46] G. Matz, D. Schafhuber, K. Grochenig, M. Hartmann, and F. Hlawatsch, "Analysis, optimization, and implementation of lowinterference wireless multicarrier systems," IEEE Transactions on Wireless Communications, vol. 6, no. 5, pp. 1921-1931, 2007.

[47] S. Das and P. Schniter, "Max-SINR ISI/ICI-shaping multicarrier communication over the doubly dispersive channel," IEEE Transactions on Signal Processing, vol. 55, no. 12, pp. 57825795, 2007.

[48] Maurice Bellanger, Personal Communication.

[49] G. W. Wornell, "Emerging applications of multirate signal processing and wavelets in digital communications," Proceedings of the IEEE, vol. 84, no. 4, pp. 586-603, 1996.

[50] L. Lin and B. Farhang-Boroujeny, "Cosine modulated multitone for very high-speed digital subscriber lines," EURASIP Journal on Applied Signal Processing, vol. 2006, Article ID 19329, 16 pages, 2006.

[51] G. Cherubini, E. Eleftheriou, and S. Olcer, "Filtered multitone modulation for VDSL," in Proceedings of the Global Telecommunications Conference (Globecom '99), vol. 2, pp. 1139-1144, Rio de Janeiro, Brazil, December 1999. 
[52] G. Cherubini, E. Eleftheriou, S. Olcer, and J. M. Cioffi, "Filter bank modulation techniques for very high-speed digital subscriber lines," IEEE Communications Magazine, vol. 38, no. 5, pp. 98-104, 2000.

[53] G. Cherubini, E. Eleftheriou, and S. Olcer, "Filtered multitone modulation for very high-speed digital subscriber lines," IEEE Journal on Selected Areas in Communications, vol. 20, no. 5, pp. 1016-1028, 2002.

[54] J. G. Proakis, Digital Communications, McGraw-Hill, New York, NY, USA, 3rd edition, 1995.

[55] A. Vahlin and N. Holte, "Optimal finite duration pulses for OFDM," IEEE Transactions on Communications, vol. 44, no. 1, pp. 10-14, 1996.

[56] C. H. (George) Yuen and B. Farhang-Boroujeny, "Sensitivity analysis of cosine modulated and offset QAM filter bank multicarrier techniques," Internal report, Wireless Lab., University of Utah, 2009.

[57] B. Boashash, Ed., Time Frequency Signal Analysis and Processing: A Comprehensive Reference, Elsevier, Oxford, UK, 2003.

[58] B. Farhang-Boroujeny, "A square-root Nyquist (M) filter design for digital communication systems," IEEE Transactions on Signal Processing, vol. 56, no. 5, pp. 2127-2132, 2008.

[59] M. Alard, "Construction of a multicarrier signal," Patent WO 96/35278, 1996.

[60] P. Siohan and C. Roche, "Cosine-modulated filterbanks based on extended Gaussian functions," IEEE Transactions on Signal Processing, vol. 48, no. 11, pp. 3052-3061, 2000.

[61] S. U. H. Qureshi, "Adaptive equalization," Proceedings of the IEEE, vol. 73, no. 9, pp. 1349-1387, 1985.

[62] B. Farhang-Boroujeny, Adaptive Filters: Theory and Applications, John Wiley \& Sons, Chichester, England, 1998.

[63] S. Haykin, Adaptive Filter Theory, Prentice Hall, Upper Saddle River, NJ, USA, 4th edition, 2001. 